
REWARD GAIN AND SHOCK REDUCTION UNDER VARIABLE INTERVAL SCHEDULES

\author{
Dave E.W. Mallpress ${ }^{1}$, Tim W. Fawcett ${ }^{1}$, John M. McNamara ${ }^{2}$ and Alasdair I. Houston ${ }^{1}$ \\ ${ }^{1}$ MODELLING ANIMAL DECISIONS (MAD) GROUP, SCHOOL OF BIOLOGICAL SCIENCES, \\ UNIVERSITY OF BRISTOL, ENGLAND \\ ${ }^{2}$ MODELLING ANIMAL DECISIONS (MAD) GROUP, SCHOOL OF MATHEMATICS, \\ UNIVERSITY OF BRISTOL, ENGLAND
}

\begin{abstract}
The relationship between positive and negative reinforcement and the symmetry of Thorndike's law of effect are unresolved issues in operant psychology. Here we show that, for a given pattern of responding on variable interval (VI) schedules with the same programmed rate of food rewards (positive reinforcement VI) or electric shocks (negative reinforcement VI), there is a fundamental mathematical equivalence between reward gain and shock reduction. We also provide the first normative account of how animals should respond on a negative VI schedule, showing that it is better to space responses evenly than to respond with a variable interresponse time (IRT). Published data from rats, however, indicate that these animals respond irregularly, often with a burst of activity immediately following a shock. While this is irrational in the experimental setting, it may represent an appropriate response to the heterogeneity of stimuli commonly encountered in natural environments. We discuss the broader implications of our analysis for understanding how animals evaluate appetitive and aversive stimuli.

Key words: law of effect, negative reinforcement, optimality, shock reduction, variable interval schedule, lever-press, rat
\end{abstract}

How animals respond to appetitive and aversive stimuli is a central focus of operant psychology and whether rewards or punishments have a stronger effect on behavior is a longstanding question in the social and animal sciences. It is often argued that these apparently distinct processes are in fact equivalent in their nature, just opposite in valence (Farley \& Fantino, 1978; Michael, 1975), but this is disputed (Magoon \& Critchfield, 2008). Here, to help clarify this debate, we provide a formal analysis of the consequences of behavior under schedules of positive and negative reinforcement.

The term reward refers to positive reinforcement, which involves an increase in the likelihood of an action due to the availability of a positive reinforcer (usually an attractive stimulus) following that action. Punishment in its technical sense involves a decrease in behavior in response to the presentation or omission of a stimulus. This is harder to study,

We thank Armando Machado, William Baum and an anonymous reviewer for their helpful and thorough comments. This work was funded by the European Research Council (Advanced Grant 250209 to AIH).

Correspondence concerning this article should be addressed to Dave Mallpress, School of Biological Sciences, University of Bristol, Woodland Road, Bristol BS8 1UG, UK (e-mail: Dave.Mallpress@bristol.ac.uk). doi: 10.1901/jeab.2012.98-355 because punishment can only be applied to actions that are already being performed and therefore "must be superimposed on behavior that is maintained by some other operation" (Magoon \& Critchfield, 2008). Because of this, much of what is known quantitatively about responses to aversive stimuli comes from research on negative reinforcement. This is defined as an increase in the likelihood of an action due to the expected delivery of an aversive stimulus, which is prevented when the action is performed.

The law of effect (Thorndike, 1905) states that actions that produce a desirable effect will be performed more often whereas those that produce an undesirable effect will be performed less often, a common finding in behavioral studies. A descriptive quantification related to this is the matching law (Herrnstein, 1961, 1970), which states that the relative rate of responding on two options matches the relative rate of positive reinforcement delivered by those options. The matching law has been extrapolated to schedules of negative reinforcement, with predictions closely matching experimental data (de Villiers, 1974; Farley \& Fantino, 1978).

It is unclear, however, whether positive and negative reinforcement should be treated as completely symmetrical within the law of effect. A requirement of this would be that 
appetitive stimuli (e.g., food rewards) and aversive stimuli (e.g., electric shocks) could be quantifiably expressed through a common metric and that reinforcers of equal and opposite magnitude could be found. Some empirical support for a symmetrical law of effect comes from experiments presenting schedules of positive and negative reinforcement concurrently to a single animal (Farley \& Fantino, 1978). These experiments found close matches to predicted choice behavior from estimates of the value of shock and food under a single currency. Moreover, some form of symmetry seems logically unavoidable when comparing the effect of presenting or removing certain stimuli: For example, the presentation of food (a positive reinforcer) can also be regarded as the removal of an imposed state of hunger (a negative reinforcer) (Baron \& Galizio, 2005; Michael, 1975). This makes it difficult not only to characterize the type of schedule imposed a priori (which would require knowing how the schedule will influence the animal's response), but even to define what counts as the stimulus (i.e., internal states of the animal). This "remains an inelegant feature of reinforcement theory" (Sidman, 2006).

The alternative to a symmetric law of effect is the differential-impact hypothesis (Critchfield \& Magoon, 2001), in which appetitive and aversive stimuli of equal magnitude differ in their ability to reinforce. Most of the experimental evidence supporting this comes from studies on humans, often using money as a comparable currency for both rewards (monetary gains) and punishments (monetary losses) (Magoon \& Critchfield, 2008; Rasmussen \& Newland, 2008; Ruddle, Bradshaw \& Szabadi, 1981). These experiments used concurrent variable interval (VI) positive and negative reinforcement schedules to determine the differential response to identicallyvalued appetitive and aversive stimuli. Similar findings have not been established for nonhuman animals, however. Some researchers have argued that even if symmetry exists, the merging of positive and negative reinforcement into a single framework is neither useful nor simplifying, and that the distinction is worth keeping for practical reasons (Iwata, 2006; Sidman, 2006).

In this paper, we focus on responses to negative reinforcement delivered under a VI schedule. There are other procedures used to study negative reinforcement, for example free operant avoidance conditioning (Sidman, 1953), but we do not consider these here because we are interested in situations in which the timing of reinforcement is unpredictable. By focusing on VI schedules, we can compare the consequences of positive and negative reinforcement directly. The negative reinforcement scenario we consider, described by de Villiers (1974), involves a test animal in an operant conditioning chamber that contains a lever (or some similar device that records responses). During the experiment, an electric shock is scheduled to be delivered to the animal at the end of each interval, which lasts a variable amount of time. Pushing the lever at any time up to the scheduled delivery of the next shock will prevent that shock. Immediately following the scheduled delivery of the shock (regardless of whether it is received or prevented), the next interval begins and all previous responses are ignored. It is thought that the reduction in shock frequency brought about by the animal's leverpushing response is sufficient to maintain that response (Herrnstein \& Hineline, 1966)

Our goal in this paper is to understand how the animal's pattern of responding under this type of schedule affects the shocks it receives, and how it can adjust the pattern of responding to achieve the optimal outcome. Thus, our focus is on how animals should respond (a normative account of behavior), rather than on how they do actually respond (a descriptive account of behavior). We start with a formal analysis of the consequences of the animal's responses for the frequency with which it is shocked under a VI negative reinforcement schedule. Mathematically, it turns out that the reduction in shock rate is equivalent to the gain rate under a VI positive reinforcement schedule. We then move on to consider the optimal pattern of responding. Behavior under VI schedules has previously been analyzed from a descriptive viewpoint, for example by modelling the feedback function between the rate of responding and the resulting rate of reinforcement (e.g., Baum, 1992; Nevin \& Baum, 1980), but little attention has been paid to how an animal should behave, that is, to optimal performance under these conditions. McNamara and Houston (1983) modelled optimal responding under a positive reinforce- 
ment VI schedule, but such a normative account is lacking for negative reinforcement. We provide this account and compare the optimality predictions with published empirical data on rats. Finally, we discuss some possible reasons why animals respond suboptimally on VI schedules.

\section{THE MODEL}

We consider the behavior of an animal exposed to a VI negative reinforcement schedule (de Villiers, 1974; Logue \& de Villiers, 1978). Shocks are scheduled as a Poisson process with rate $\lambda$, such that if the animal does not respond it will be shocked on average $\lambda$ times per unit time. This means that the interval between scheduled shocks has an exponential distribution with a mean of $1 / \lambda$. If the animal performs a response (e.g., pushes a lever), it prevents the occurrence of the next shock. For example, if three shocks are scheduled between two consecutive responses, only the last two of those shocks will actually be received.

First we consider the case in which the animal responds with a constant time between responses. This interresponse time (IRT) is denoted by $t$. The expected number of shocks scheduled (not received) between two consecutive responses is then $\lambda t$. If at least one shock is scheduled during the IRT, which happens with probability $1-e^{-\lambda t}$, the animal will receive one less shock than the scheduled number $\lambda t$, because the first shock is prevented by its last response. Therefore the expected reduction in the number of shocks is $1 \times\left(1-e^{-\lambda t}\right)$. Dividing this by the IRT gives us the expected rate of shock reduction, $S(t)$ :

$$
S(t)=\frac{1-e^{-\lambda t}}{t}
$$

At high rates of responding (where $t$ approaches 0$), S(t)$ approaches $\lambda$ because the animal prevents almost every shock. If the animal responds rarely (i.e., $t$ becomes large), the rate of shock reduction approaches zero.

We can compare this equation with the effect of responding with a constant IRT to obtain positive reinforcement. Under a VI positive reinforcement schedule (Fleshler \& Hoffman, 1962), rewards become available (are 'set up') at rate $\lambda$, such that the time for a given reward to be set up has an exponential distribution with mean $1 / \lambda$. Until the animal makes a response, however, it receives no information on whether a reward has been set up. Responses prior to the set-up time have no effect, but the animal's first response after the set-up time results in collection of the reward and the beginning of a new (variable) interval. In the standard positive reinforcement VI schedule when a reward is set up the VI clock stops, and it restarts only when the (reinforced) response occurs. Note that, due to the memoryless property of the exponential distribution (Grimmet \& Stirzaker, 2001), this stopping of the clock does not affect the expected time to the set-up of the next reward once a response has been made.

Analogously to the negative reinforcement VI schedule, the probability that a reward is set up between two consecutive responses is $1-$ $e^{-\lambda t}$ (McNamara \& Houston, 1983). The expected number of rewards gained per unit time, $R_{r}(t)$, is therefore

$$
R_{r}(t)=\frac{1-e^{-\lambda t}}{t}
$$

where $\lambda$ is the rate at which rewards are set up, analogous to the scheduled shock rate in the VI negative reinforcement procedure above. Comparing equations (1) and (2), it is immediately apparent that the rate of reward gain, $R_{r}(t)$, under a VI positive reinforcement schedule, is identical to the rate of shock reduction, $S(t)$, under a VI negative reinforcement schedule with the same rate parameter $\lambda$. This means that for any given pattern of responding with a constant IRT, the chance that each response produces a desirable outcome (whether gaining a food reward or preventing a shock) is the same for VI schedules of negative or positive reinforcement.

\section{Equivalence of Positive and Negative Reinforcement}

This equivalence relationship can be extended to any variable pattern of responding where the timing of the animal's responses is independent of the timing of the reinforcing stimuli. Under a negative reinforcement schedule, the animal prevents the first of any shocks that are scheduled between two consecutive responses. It therefore prevents one shock if and only if a new shock is scheduled during the time between those responses. Under a positive reinforcement schedule, the 
animal receives one reward when making a response if and only if a new reward has been set up during the time since its last response. Thus, regardless of the pattern of responding, the chance of preventing a shock or receiving a reward is determined by whether or not a new stimulus was scheduled between two consecutive responses. This implies that, for the same pattern of responding and the same programmed reinforcement rate $\lambda$, the rate of shock reduction in a negative reinforcement VI schedule equals the rate of reward gain in a positive reinforcement VI schedule. It is important to note, however, that this equivalence only holds when the timing of responses is independent of the timing of the reinforcing stimuli. If the animal's responses depend on the receipt or absence of individual rewards or shocks, the probabilities of being reinforced for those responses will differ between the positive and negative cases.

\section{Optimal Responding}

We now consider the optimal pattern of responding under a VI negative reinforcement schedule (for optimal responding under a VI positive reinforcement schedule, see McNamara \& Houston, 1983; Staddon \& Motheral, 1978). We assume that receiving a shock and making a response are both costly to the animal. Electric shocks may induce various sympathetic processes that deplete energy, and in extreme cases can cause longterm physiological damage or even death. Making a response (e.g., pressing a lever) must also entail some non-negligible energetic cost, because otherwise we would expect animals to respond at the maximum possible rate just to avoid a shock. In general, it seems likely that the cost of receiving a shock, here denoted by $c_{S}$, is considerably greater than the energetic cost of responding, denoted by $c_{L}$. To reflect this we use $c_{L}=\alpha c_{S}$ with $\alpha<1$, where the factor $\alpha$ ensures that the two currencies are commensurable and can easily be translated within our model. Costs are assumed to be additive and because the animal is in an operant chamber, we also assume that there are no opportunity costs. For a given rate of responding, $1 / t$, the expected rate of shocks received by the animal is equal to the scheduled rate $\lambda$ minus the rate of shock reduction $S(t)$. The total expected cost per unit time, $C(t)$, is therefore

$$
\begin{gathered}
C(t)=(\lambda-S(t)) c_{S}+\frac{1}{t} c_{L} \\
=\frac{\left(\lambda t+e^{-\lambda t}-1+\alpha\right) c_{S}}{t}
\end{gathered}
$$

We assume that natural selection has favored animals that minimize the total costs that they incur, such that an animal behaving optimally will minimize $C(t)$. The optimal time between responses, $t^{*}$, is found by differentiating equation (3) with respect to $t$ and setting the result equal to zero, which simplifies to

$$
1-\alpha=e^{-\lambda t^{*}}\left(1+\lambda t^{*}\right)
$$

Numerical solutions for $t^{*}$ under four different scheduled shock rates $(\lambda)$ are plotted in Figure 1, for $\alpha=0.1$ (i.e., shocks are 10 times as costly as responses). There is a tradeoff between responding too frequently (avoiding shocks but expending too much energy in responding) and responding too infrequently (requiring little energy but resulting in a high received shock rate). As the scheduled rate of reinforcement $(\lambda)$ increases, the optimal behavior involves responding more often.

Optimal behavior can also be identified graphically, by plotting the received shock rate $R_{s}(t)$ against the response rate $1 / t$ (Figure 2, black curve). This curve captures the fundamental tradeoff between minimizing the costs of being shocked and minimizing the costs of responding. The optimal response rate, $1 / t^{*}$, occurs at the point where a straight line with slope $-\alpha$ (gray line) is tangent to the response curve, since this is where the total expected cost per unit time is minimized.

\section{Constant versus Variable IRT}

Instead of simply responding at regular intervals, the animal may respond with a variable IRT. Following Staddon \& Motheral (1978) and McNamara \& Houston (1983), we model the pattern of responding as a Poisson process. We use the random variable $T$ to represent the (variable) IRT, which follows an exponential distribution with mean $E(T)=t$, giving an overall response rate of $1 / t$.

We are now dealing with two independent Poisson processes, one describing the pattern 


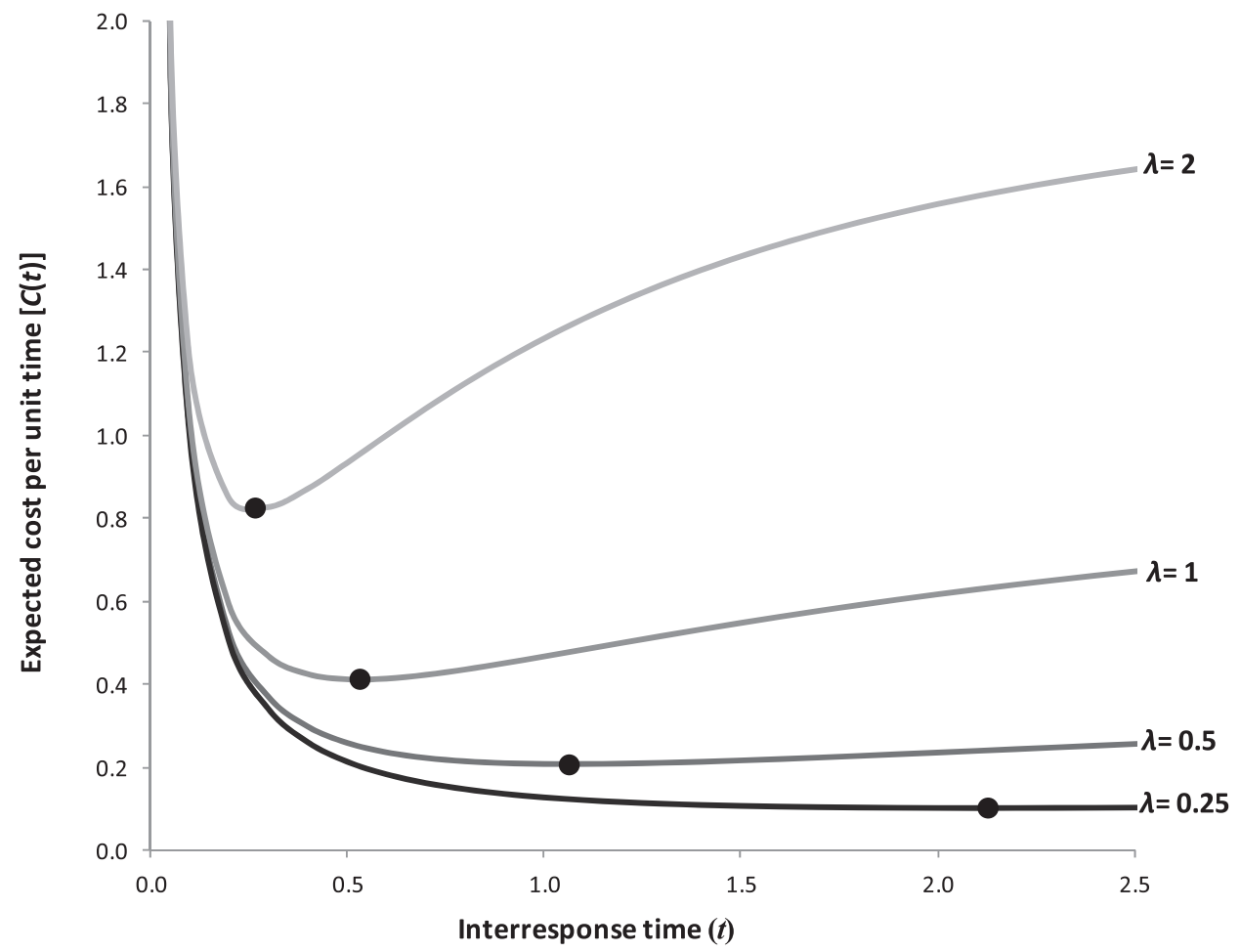

Fig. 1. The expected total cost per unit time $[C(t)]$ incurred by an animal responding with a constant IRT $(t)$ on a VI negative reinforcement schedule. Shocks are assumed to be 10 times more costly to the animal than making a response $(\alpha=0.1)$. For each scheduled reinforcement rate $\lambda$, the optimal IRT $t^{*}$ (indicated by the black circles) minimizes the total cost incurred.

of scheduled shocks (with rate $\lambda$ ) and the other describing the pattern of responding (with rate $1 / t$ ). A shock is prevented in those cases where a response happens before the shock is scheduled. According to standard probability theory (Grimmet \& Stirzaker, 2001), the probability of this ordering of events (response before shock) is the rate of responding divided by the sum of the two rates, $(1 / t) /(\lambda+1 / t)$, which simplifies to $1 /$ $(\lambda t+1)$. To obtain the rate of shock reduction, $S(T)$, we multiply this probability of preventing a shock by $\lambda$, the rate of scheduled shocks:

$$
S(T)=\frac{\lambda}{\lambda t+1}
$$

By comparing equation (5) with equation (1), one can show that the expected rate of shock reduction will always be higher when responding with a constant IRT $(t)$ than with exponentially distributed IRTs $(T)$, given that the long-term response rate is the same in both cases. This is because $\left(1-e^{-\lambda t}\right) / t \geq \lambda /(\lambda t$
+1 ) for all $\lambda, t>0$. The cost of responding per unit time is the same in both cases, which implies that responding with a constant IRT will result in lower total costs than responding at exponentially distributed intervals.

The superiority of a constant IRT can be shown more generally using Jensen's inequality, as illustrated in Figure 3. The probability of preventing a shock as a function of time since the last response is a concave-down function, in that it is increasing but decelerating. Jensen's inequality states that, because of this concave-down form, the mean of the values of the function at two different points $(t-\varepsilon$ and $t+\varepsilon)$ is less than the value of the function evaluated at the mean of those two points $(t)$. This implies that an animal on a VI schedule will on average always perform at least as well when responding with a constant IRT than a variable IRT with the same mean (Figure 3; see also McNamara \& Houston, 1983). 


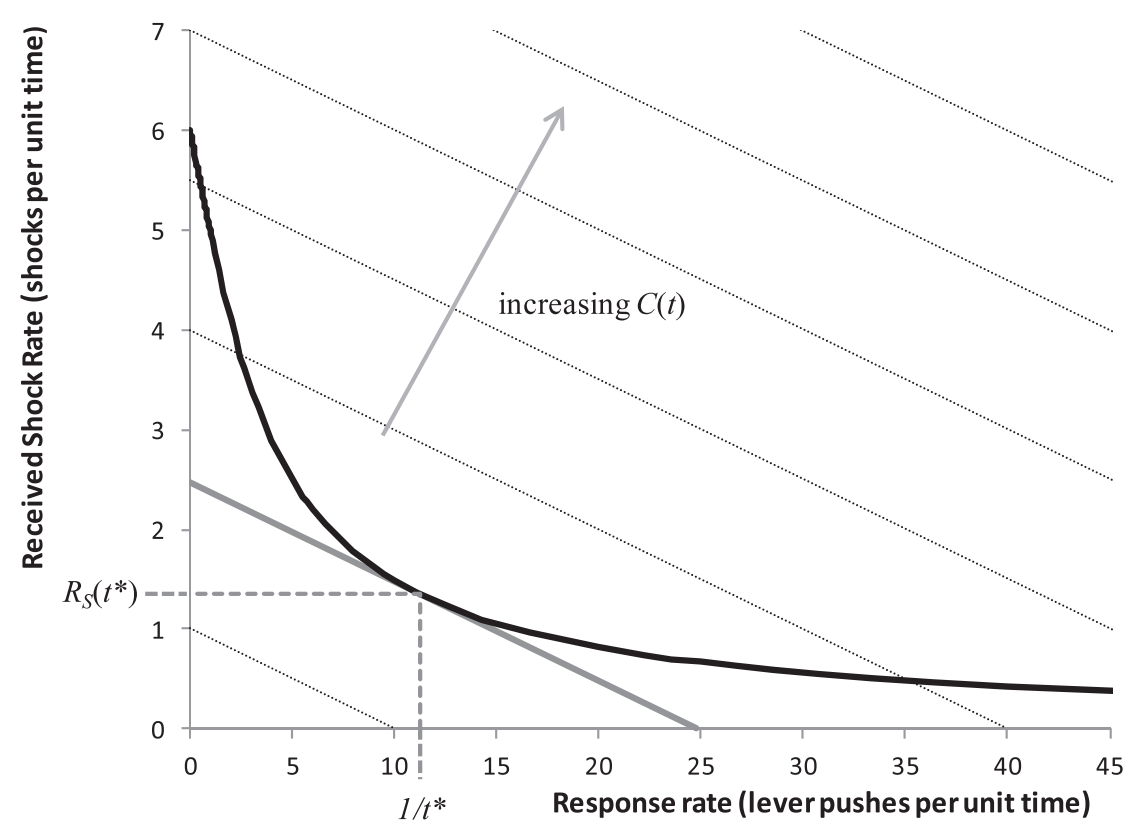

Fig. 2. Graphical method for identifying the optimal response rate on a VI negative reinforcement schedule with scheduled shock rate $\lambda=6$, for an animal responding at regular intervals. The black curve plots the expected received shock rate $(\lambda-S(t))$ against the response rate $1 / t$. Each of the diagonal lines has slope $-\alpha$ (representing the marginal rate of substitution) and connects points for which the total expected cost per unit time, $C(t)$, is constant; $C(t)$ increases in the direction shown by the arrow. The tangent to the curve identifies the point at which the total expected cost per unit time is minimized, and therefore behavior is optimal.

\section{Comparison with Positive Reinforcement}

Optimal behavior under a VI positive reinforcement schedule was modelled by McNamara \& Houston (1983). To maximize its net reward rate, the animal faces a tradeoff between the cost of responding and the value of the reward. Since a constant IRT outperforms a variable IRT (McNamara \& Houston), here we will only consider the former case, where the animal makes a response every $t$ time units. Following from equation (2) and denoting the value of each reward item as $v_{r}$ and the cost of making a response as $c_{L}=\beta v_{r}$, the expected net reward rate, $U(t)$, is

$$
\begin{aligned}
& U(t)=R_{r}(t) v_{r}-\frac{c_{L}}{t} \\
& =\frac{\left(1-e^{-\lambda t}-\beta\right) v_{r}}{t}
\end{aligned}
$$

where $\beta$ is a parameter scaling the cost of making a response relative to the value of a reward. The optimal time between responses, $t^{*}$, is found by differentiating equation (6) with respect to $t$ and then setting the result equal to zero, which simplifies to

$$
1-\beta=e^{-\lambda t^{*}}\left(1+\lambda t^{*}\right)
$$

Note that this solution is mathematically equivalent to the optimal solution found under VI negative reinforcement (equation $4)$; the only difference is in the scaling factors $\alpha$ and $\beta$, which convert between two costs in the case of negative reinforcement and between a cost and a benefit in the case of positive reinforcement.

\section{What the Empirical Data Show}

We now relate the insights from our optimality model to published empirical data from rats on a VI schedule of negative reinforcement. de Villiers (1974) investigated the behavior of 4 rats on a variety of VI schedules (average scheduled shock rate ranging from $15 \mathrm{~s}$ to $60 \mathrm{~s}$ ). In Figure 4 we plot the behavior of each rat together with the curves relating received shock rate to response rate, for both a constant IRT (black curves) and a variable IRT based on a Poisson process (gray curves). 


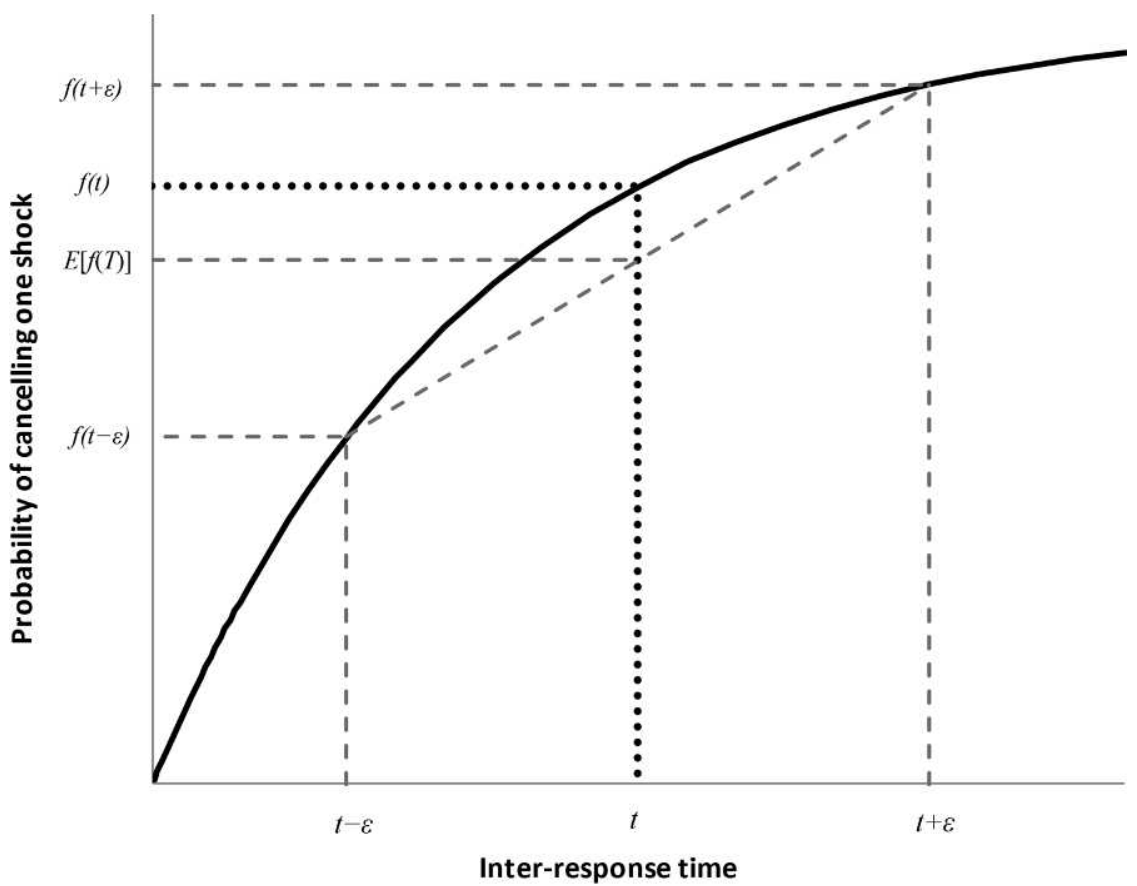

Fig. 3. A graphical representation of Jensen's inequality. The solid line shows the probability of cancelling one shock as a function of IRT length. An animal responding with IRTs of constant length $T=t$ would cancel one shock with probability $f(t)$ (dotted lines). An animal responding with IRTs of variable length but with the same average-for example, IRTs equal to $T=t-\varepsilon$ or $T=t+\varepsilon$, each with probability one half so that $E(T)=t$ (dashed lines)-would cancel one shock with probabilities $f(t-\varepsilon)$ and $f(t+\varepsilon)$, respectively. Due to the concave-down curvature of the solid line, the probability of cancelling one shock by responding with a constant IRT is always greater than the average probability of cancelling one shock by responding with a variable IRT, that is, $f(t)>E[f(T)]$

Individual rats differed consistently in their response rates (GLM, effect of rat identity: $\left.F_{3,22}=81.23, p<0.001\right)$. It is clear that the rats were not behaving optimally because they did not space their responses evenly: Their received shock rate was consistently higher than that expected for the same mean response rate when responding with a constant IRT. Their received shock rate was also typically higher than that expected for variable IRTs following a Poisson process. As noted by de Villiers (1974), many of the rats reacted with short bursts of rapid responding immediately following a shock.

\section{DISCUSSION}

\section{Positive versus Negative Reinforcement}

The first and most prominent result that emerges from our analysis is the fundamental equivalence of the consequences of responding for negative reinforcement and positive reinforcement. For a given rate of scheduled reinforcement $(\lambda)$ and a given pattern of responding, the rate of reward gain on the VI positive reinforcement schedule (equation 2 ) is exactly the same as the rate of shock reduction on the VI negative reinforcement schedule (equation 1). This generalized relationship holds only if the pattern of responding is independent of the scheduled reinforcing stimuli. If the animal responds directly to a given reinforcement event, the equivalence breaks down.

It follows from this that the theoretically optimal behavior is the same under positive and negative VI reinforcement schedules. An animal on a VI schedule should therefore respond in the same way to positive and negative stimuli that have equivalent but opposite effect on fitness. These normative predictions come from a purely functional approach, based on the assumption that an animal's behavior has been shaped by natural selection. They are not mechanistic and in no way attempt to characterize the underlying 

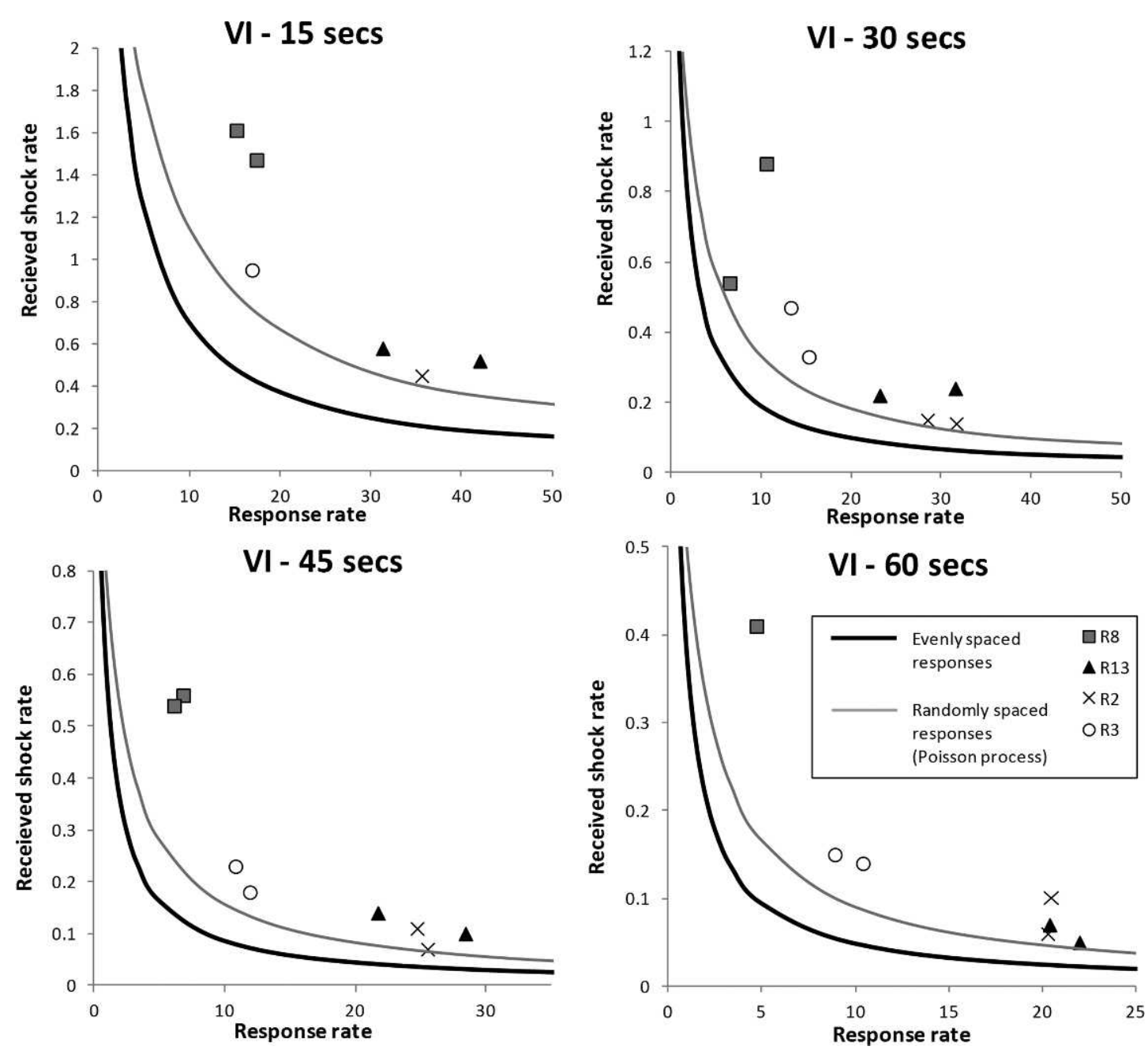

Fig, 4. The response rates (number of responses per minute) and received shock rates (number of shocks received per minute) of 4 rats on different VI schedules, as reported by de Villiers (1974). For comparison, the curves show the expected received shock rate $(\lambda-S[T])$ for an animal whose responses are evenly spaced in time (black curve) or randomly distributed according to a Poisson process (gray curve). Each rat was tested twice on each schedule, except for rats R2 and R3 on the VI 15-s schedule (tested only once) and R8 on the VI 60-s schedule (tested only once).

physical and psychological processes that could lead to the optimal (or suboptimal) behavior.

This equivalence in the theory again raises the question of whether the law of effect is symmetrical; that is, can appetitive and aversive events be converted into a single currency and thus can tradeoffs be summarized by a single value? Abolishing the distinction between positive reinforcement (presentation of a stimulus) and negative reinforcement (removal of a stimulus) has been both advocated (Baron and Galizio, 2005; Michael, 1975) and opposed (Iwata, 2006; Sidman, 2006). Skinner himself noted that "the cessation of a positive reinforcement acts as a negative, the cessation of a negative acts as a positive" (Skinner, 1938, p 66). This interpretation would suggest that positive and negative reinforcement are just two components of a one-dimensional spectrum, with every appetitive state corresponding to the removal of an aversive state (Baron \& Galizo, 2005). There is some evidence in support of this hypothesis from reinforcement experiments in animals (Baum, 1973; de Villiers, 1974; Farley \& Fantino, 1978; Leiten- 
berg, 1965). For example, Farley and Fantino utilized the matching law to obtain estimated values for a shock per minute (in a concurrent negative VI schedule) in terms of negative food units (in a concurrent positive VI schedule) using the same individuals. These predictions were then shown to match the empirical results of a concurrent positive-VI negative-VI schedule.

Empirical work in humans supports an alternative interpretation. Evidence from concurrent VI reinforcement studies (Magoon \& Critchfield, 2008; Rasmussen \& Newland, 2008) and from prospect theory (Tversky \& Kahneman, 1992) supports an asymmetry of gains and losses within a common metric of money. Such evidence does not necessarily mean, however, that reward and punishment are dealt with through independent systems in the brain. Instead, framing effects, anchoring and other cognitive and social biases may generate asymmetries in perception in human studies (Caverni, Fabre \& Gonzalez, 1990). Although many other animals share a range of cognitive biases with humans, it is difficult to extrapolate these results to other species because nonhuman animals have no general currency in which both appetitive and aversive stimuli can be presented.

Due to differences in the procedures between positive and negative VI reinforcement schedules, there are some substantial asymmetries in the information that the animal obtains during the schedule (Magoon \& Critchfield, 2008). In positive reinforcement, the animal receives no information about whether a reward has been set up until it makes a response, and the consequence of each response informs the animal about whether or not a reward was available. In contrast, under negative reinforcement the animal receives information about when a shock is scheduled if it fails to respond in time; a successful response (one which prevents the next shock) is not signalled to the animal at all, and is indistinguishable from any subsequent, ineffective responses made before the next interval begins. This informational asymmetry is not captured by our normative model, but it may be important in understanding cases where animals respond differently on the two types of schedule. In VI positive reinforcement, the reward can be easily attributed to the response because it is delivered immedi- ately after the response. In VI negative reinforcement, in contrast, the time between the response and the delivery of a shock is variable, which may make it more difficult to form associations between the two.

With respect to the delay between a response and its consequence, a more direct positive equivalent to the negative reinforcement VI schedule would be one in which rewards are not delivered immediately after a successful response, but after an exponentially distributed interval since the last reward. Specifically, rewards could be scheduled as a Poisson process and only delivered if a response is made during the interval between two consecutive scheduled rewards. Both the negative and positive VI schedules can then be viewed as a Poisson stream of scheduled stimuli (shocks or rewards), with the animal having to respond at least once between two consecutive stimuli in order to achieve the desirable outcome (receiving a reward or cancelling a shock). In both cases, the delay between the reinforced response and the reinforcer itself is variable, which potentially makes it harder for the animal to form associations between the two. To our knowledge, this type of positive VI schedule has never been used. The optimal behavior under such conditions would be to respond immediately following the delivery of each reward, thereby guaranteeing receipt of all available rewards with only one response necessary for each. On the negative reinforcement schedule such an efficient outcome is not possible, since the animal is only informed of the start of a new interval if it failed to respond during the previous interval. An animal that only responds immediately following a shock would cancel alternate shocks, leading to an overall shock reduction of $50 \%$.

The positive and negative schedules could hypothetically be made symmetric in human studies, by indicating a 'missed reward' at the end of intervals in positive reinforcement and indicating a 'shock removed' following a successful response in negative reinforcement. However, the essence of the VI schedule would be lost, because (similar to the case above) responding once at the start of each new interval would become the optimal behavior. It therefore seems that these procedural asymmetries are a fundamental part of the experimental design. This does not, however, 
rule out the possibility of evaluating aversive and appetitive events through comparison with punishment experiments. For example, behaviors maintained by positive reinforcers can be reduced by superimposing punishment onto a standard positive reinforcement schedule (Bouzas, 1978; Critchfield, Paletz, MacAleese \& Newland, 2003; Rachlin \& Herrnstein, 1969; Wylie \& Grossman, 1988). By incorporating a punishing stimulus incrementally alongside the reinforcer, the point at which the animal becomes indifferent to performing the response (and therefore ceases to respond) would theoretically indicate when the stimuli are of equal and opposite value (ignoring the costs of responding; see Rachlin \& Herrnstein).

\section{Optimal versus Observed Behavior}

The ecological relevance of positive reinforcement in the form of food is well understood. Positive reinforcement schedules can be seen as a simple energetic trade-off, where an energetic cost of responding (e.g., pressing a lever) is directly commensurable to energetic gain from food received. By contrast, we have a poor understanding of the ecological relevance of negative reinforcement, where no simple trade-off can be made between the energy used in responding and the negative consequences of an aversive stimulus such as an electric shock, which may be only partly energetic and are difficult to quantify. In our models we used the parameter $\alpha$ to denote the ratio between the cost of responding and the cost of receiving a shock. This term, known in economics as the marginal rate of substitution, has been effectively applied in behavioral ecology to compare the benefit of gaining energy with the cost of being killed (Caraco, 1979; Houston \& McNamara, 1989, 1999). The 4 rats tested by de Villiers (1974) showed consistently different response rates across schedules. In the context of our model, this may indicate different subjective weightings of the negative consequences of a shock relative to the energetic cost of responding (i.e., different marginal rates of substitution, $\alpha$ values).

A key prediction from our optimality analysis is that an animal under a VI negative reinforcement schedule should respond with a constant IRT. Its responses should be independent of any shocks it receives between responses and there should be no change in rate due to the recent presentation or absence of a shock. de Villiers (1974) reported that the rats did generally respond at steady rates, though a quantification of the variability in their response rates was not given. From our analysis it is clear that the rats were not behaving in an optimal way, receiving at least twice the number of shocks they would have received if they had spaced their responses evenly. Even though de Villiers's rats were exposed to the test conditions for many sessions before results were recorded, there was still an increased response rate immediately following a shock. Similar experiments have found sporadic bursts in animals' response rates in both negative (Herrnstein \& Hineline, 1966; Sidman, 1958) and positive (Shull, Gaynor \& Grimes, 2001; Shull \& Grimes, 2003) reinforcement setups. Bursts of responding like this have been modelled descriptively using feedback functions to show how the rate of response affects the rate of reinforcement, with a good fit to the empirical data (Baum, 1992).

Timberlake (1993) suggests that reinforcement alone is insufficient to explain animal behavior and that ecological (as well as regulatory and cognitive) causal factors need to be taken into account for a more complete understanding of behavior. This approach emphasizes that learning is constrained by unlearned, species-typical propensities to behave in ways that were appropriate for the animal's ancestral environment. Though not optimal in an artificial VI schedule, sudden bursts of responding immediately after an aversive stimulus could be adaptive in a more heterogeneous environment in which stimuli are clustered in space or time. For example, in natural environments the presence of a predator at one time has a strong positive correlation with the presence of a predator soon afterwards. Similarly, food and mates are often sparsely distributed in patches and bad weather now is a good predictor of bad weather in 20 minutes' time. The VI schedules we have analyzed fail to capture such heterogeneity. Programmed shocks on these schedules occur as a Poisson process, but very few natural phenomena at the macro level follow a Poisson process with events being completely independent of one another.

Electric shocks have been used as the standard aversive stimulus for behavioral ex- 
periments for the best part of the last 100 years. There are good pragmatic reasons for this, such as the ability to keep shock intensity constant throughout trials and systematically vary the intensity (quantitatively) across trials without modifying the apparatus. However, this approach has made it difficult to extrapolate findings beyond the conditioning chamber and into the environment to which the animal is adapted (Fanselow, Lester \& Helmstetter, 1988). Do electric shocks hold any ecological relevance for the animal being studied? Some operant experiments treat behavior as avoidance in an energy-predation tradeoff, with the animal expending costs of being vigilant (or forgoing foraging opportunities) to reap the benefits of a reduced risk of being predated (Bolles, 1970; Fanselow et al.). If the shock is simply a stimulus that induces pain, it acts as an indicator of somatic damage which the animal should escape from and in the future avoid, which might explain a burst of responding following the shock. On the other hand, postshock increases in the rate of responding seem unlikely to be escape responses because the shock will have already terminated by the time the animal starts responding (Boren, 1961). Instead, exposure to the ambiguous painful stimulus may induce a specific behavioral repertoire associated with pain, which includes activities that were historically relevant to surviving pain-related stimuli (Baum, 2012). Another possible interpretation of the effect of a shock is that it causes an acute stress reaction in response to a perceived potential predator. Under these conditions, one might expect the focal animal to exhibit more freezing and vigilance behavior. This seems more plausible for shocks of a low current and short duration, which probably induce an affective state of surprise and anxiety more than they generate pain.

\section{Limitations and Extensions}

For the sake of mathematical simplicity, we assumed that gains and costs are constant. Under some circumstances, however, costs may change depending on the costs already incurred. For example, the first few shocks may be fairly harmless to the animal, but a large number could put the animal under excessive stress or become life-threatening. In other situations costs may be subject to diminishing returns-the first few shocks might be very costly, but as the animal habituates to them the costs of later shocks are lower than earlier shocks. The same principle might apply to costs of lever pushing, with later pushes being more costly than earlier ones due to muscle fatigue, or perhaps less costly because of learning to push the lever more efficiently (Brener \& Mitchell, 1989). Further work is needed to predict how animals should respond in these interesting and biologically realistic cases of nonlinearity, where the marginal rate of substitution changes with state (cf. the energy-predation tradeoff, in which the marginal rate of substitution of predation risk for energy gain is dependent on current energy reserves; Houston \&McNamara 1999).

We have taken a normative approach here to elucidate the economics of responding on a VI schedule, but a more mechanistic approach could be instructive. As mentioned above, the amount of time that has elapsed between response and reinforcer may affect how easily an animal can form associations between the two. One possible approach would be to directly model changes in the strength of learned associations between response and reinforcer, assuming some specific pattern of decline in the strength as the temporal separation increases. Killeen (2011) has proposed one such model, based on the idea that the number of competing stimuli increases exponentially with the delay between response and reinforcer, and hence weakens reinforcement. It would be interesting to apply this kind of approach to the VI schedules we have considered here. Temporal proximity plays a role in the ability to attribute a consequence to a previous action (Chung \& Herrnstein, 1967; Renner, 1964; Tarpy \& Sawabini, 1974) so mechanistic models of learning should include this.

\section{REFERENCES}

Baron, A., \& Galizo, M. (2005). Positive and negative reinforcement: Should the distinction be preserved? The Behavioral Analyst, 28, 85-98.

Baum, W.M. (1973). Time allocation and negative reinforcement. Journal of the Experimental Analysis of Behavior, 20, 313-322.

Baum, W.M. (1992). In search of feedback functions for variable-interval schedules. Journal of the Experimental Analysis of Behavior, 57, 365-375.

Baum, W.M. (2012). Rethinking reinforcement: Allocation, induction and contingency. Journal of the Experimental Analysis of Behavior, 97, 101-124. 
Bolles, R.C. (1970). Species-specific defence reactions and avoidance learning. Psychological Review, 77, 32-48.

Boren, J.J. (1961). Isolation of post-shock responding in a free operant avoidance procedure. Psychological Reports, 9, 265-266.

Bouzas, A. (1978). The relative law of effect: Effects of shock intensity on response strength in multiple schedules. Journal of the Experimental Analysis of Behavior, 30, 307-314.

Brener, J., \& Mitchell, S. (1989). Changes in energy expenditure and work during response acquisition in rats. Journal of Experimental Psychology: Animal Behavior Processses, 15, 166-175.

Caraco, T. (1979). Time budgeting and group size: a theory. Ecology, 60, 611-617.

Caverni, J. P., Fabre, J. M., \& Gonzalez, M. (1990). Cognitive biases, advances in psychology: 68. Amsterdam: Elsevier Science Publishers B.V.

Chung, S.-H., \& Herrnstein R.J. (1967). Choice and delay of reinforcement. Journal of the Experimental Analysis of Behavior, 10, 67-74.

Critchfield, T.S., \& Magoon, M.A. (2001). On the differential impact of positive and negative reinforcement. Experimental Analysis of Human Behavior Bulletin, 19, 16-18.

Critchfield, T.S., Paletz, E.M., MacAleese, K.R., \& Newland, M.C. (2003). Punishment in human choice: Direct or competitive suppression? Journal of the Experimental Analysis of Behavior, 80, 1-27

de Villiers, P.A. (1974). The law of effect and avoidance: a quantitative relationship between response rate and shock-frequency reduction. Journal of the Experimental Analysis of Behavior, 21, 223-235.

Fanselow, M.S., Lester, L.S., \& Helmstetter, F.J. (1988). Changes in feeding and foraging patterns as an antipredator defensive strategy: a laboratory simulation using aversive stimulation in a closed economy. Journal of the Experimental Analysis of Behavior, 50, 361374.

Farley, J., \& Fantino, E. (1978). The symmetrical law of effect and the matching relation in choice behavior. Journal of the Experimental Analysis of Behavior, 29, 3760.

Fleshler, M., \& Hoffman, H. S. (1962). A progression for generating variable-interval schedules. Journal of the Experimental Analysis of Behavior, 5, 529-530.

Grimmett, G.R., and Stirzaker, D.R. (2001). Probability and random processes (3rd edition). Oxford: Oxford University Press.

Herrnstein R.J. (1961). Relative and absolute strength of response as a function of frequency of reinforcement. Journal of the Experimental Analysis of Behavior, 4, 267-272.

Herrnstein R.J. (1970). On the law of effect. Journal of the Experimental Analysis of Behavior, 13, 243-266.

Herrnstein, R.J., \& Hineline, P.N. (1966). Negative reinforcement as shock-frequency reduction. Journal of the Experimental Analysis of Behavior, 9, 421-430.

Houston, A., \& McNamara, J. (1989). The value of food: effects of open and closed economies. Animal Behavior, 37, 546-562.

Houston, A.I., \& McNamara, J.M. (1999). Models of adaptive behavior: An approach based on state. Cambridge: Cambridge University Press.

Iwata, B.A. (2006). On the distinction between positive and negative reinforcement. The Behavior Analyst, 29, 121-123.
Killeen, P.R. (2011). Models of trace delay, eligibility for reinforcement, and delay of reinforcement gradients, from exponential to hyperboloid. Behavioral Processes, $87,57-63$.

Leitenberg, H. (1965). Is time out from positive reinforcement an aversive event? A review of the experimental literature. Psychological Bulletin. 64, 428-441.

Logue, A.W., \& de Villiers, P.A. (1978). Matching in concurrent variable-interval avoidance schedules. Journal of the Experimental Analysis of Behavior, 29, 61-66.

Magoon, M.A., \& Critchfield, T.S. (2008). Concurrent schedules of positive and negative reinforcement: differential-impact and differential-outcomes hypotheses. Journal of the Experimental Analysis of Behavior, 90, $1-22$.

McNamara, J.M., \& Houston A.I. (1983). Optimal responding on variable interval schedules. Behavior Analysis Letters, 3, 157-170.

Michael, J. (1975). Positive and negative reinforcement, a distinction that is no longer necessary; or a better way to talk about bad things. Behaviorism, 3, 33-44.

Nevin, J.A., \& Baum, W.M. (1980). Feedback functions for variable-interval reinforcement. Journal of the Experimental Analysis of Behavior, 34, 207-217.

Rachlin, H., \& Herrnstein, R.J. (1969). Hedonism revisited: On the negative law of effect. In B. Campbell \& R. M. Church (Eds.), Punishment and aversive behaviour (pp. 83-109). New York: Appleton-Century-Crofts.

Rasmussen, E.B., \& Newland, M.C. (2008). Asymmetry of reinforcement and punishment in human choice. Journal of the Experimental Analysis of Behavior, 98, 157167.

Renner, E.K. (1964). Delay of reinforcement: A historical review. Psychological Bulletin, 61, 341-361.

Ruddle, H.V., Bradshaw, C.M., \& Szabadi, E. (1981). Performance of humans in variable-interval avoidance schedules programmed singly and concurrently with variable schedules of positive reinforcement. Quarterly Journal of Experimental Psychology, 33, 213-226.

Shull, R.L., Gaynor, S.T., \& Grimes, J.A. (2001). Response rate viewed as engagement bouts: Effects of relative reinforcement and schedule type. Journal of the Experimental Analysis of Behavior, 75, 247-274.

Shull, R.L., \& Grimes, J.A. (2003). Bouts of responding from variable-interval reinforcement of lever pressing in rats. Journal of the Experimental Analysis of Behavior, 80, 159-171.

Sidman, M. (1953). Avoidance conditioning with brief shock and no exteroceptive warning. Science, 118, $157-158$.

Sidman, M. (1958). Some notes on "bursts" in freeoperant avoidance experiments. Journal of the Experimental Analysis of Behavior, 1, 167-72.

Sidman, M. (2006). The distinction between positive and negative reinforcement: Some additional considerations. The Behavior Analyst. 29, 135-139.

Skinner, B.F. (1938). The behavior of organisms, An experimental analysis. NY: D. Appleton-Century Company Inc.

Staddon, J.E.R., \& Motheral, S. (1978). On matching and maximizing in operant choice experiments. Psychological Review, 85, 436-444.

Tarpy, R.M., \& Sawabini F.L. (1974). Reinforcement delay: A selective review of the last decade. Psychological Bulletin, 81, 984-997. 
Thorndike, E.L. (1905). The elements of psychology. NY: A.G. Wylie, A.M., \& Grossman, J.A. (1988). Response reduction Seiler.

Timberlake, W. (1993). Behavior systems and reinforcement: An integrative approach. Journal of the Experimental Analysis of Behavior, 60, 105-128.

Tversky, A., \& Kahneman, D. (1992). Advances in prospect theory: Cumulative representation of uncertainty. Journal of Risk and Uncertainty, 5, 297-323. through the superimposition of continuous reinforcement: a systematic replication. Journal of Applied Behavior Analysis, 21, 201-206.

Received: March 12, 2012

Final Acceptance: August 20, 2012 